\title{
Pleural resection and pleural infusion chemotherapy for therapy of malignant pleural mesothelioma
}

\author{
J. Zhang ${ }^{1}$, Q. Zhang ${ }^{2}$, W. Zhao ${ }^{1}$, J. Duan ${ }^{1}$ and T. Huang ${ }^{1}$ \\ ${ }^{1}$ Department of Thoracic Surgery, \\ The First Affiliated Hospital of Kunming Medical University, \\ Kunming, Yunnan, China \\ ${ }^{2}$ Department of Pharmacy, \\ The First Affiliated Hospital of Kunming Medical University, \\ Kunming, Yunnan, China \\ Corresponding author: T. Huang \\ E-mail: jzcn2000@163.com
}

Genet. Mol. Res. 13 (1): 483-489 (2014)

Received August 23, 2013

Accepted October 26, 2013

Published January 21, 2014

DOI http://dx.doi.org/10.4238/2014.January.21.17

\begin{abstract}
The aim of this study was to evaluate effective treatment methods of malignant pleural mesothelioma (MPM). Twenty-three patients with MPM were cured by pleural infusion chemotherapy after surgery. Median survival time and the 1-, 2-, and 3-year survival rates were analyzed on the basis of follow-up. Median survival time of all patients was 15 months (range: 3 to 89 months); the 1-, 2-, and 3 -year survival rates were $69.6,43.5$, and $13.0 \%$, respectively. The 1-year survival rates of patients in stages I, II, and III were 83.3, 62.5, and $33.3 \%$, respectively, the 2 -year survival rates were $50,37.5$, and $33.3 \%$, respectively, and the 3 -year survival rates were 34,23 , and $0 \%$, respectively. Surgery-oriented comprehensive treatment was adopted for MPM, which could improve the prognosis and life quality of patients to some extent.
\end{abstract}

Key words: Pleural tumor; Surgical treatment; Chemotherapy; Tumors; Local infusion 


\section{INTRODUCTION}

Malignant pleural mesothelioma (MPM) is a rare tumor originating in mesothelial cells. It often occurs in parietal pleura or diaphragmatic pleura and involves visceral pleura, pericardium, and mediastinal tissue. It can also be found in the peritoneum. Its degree of malignancy is relatively high. The incidence rate is 0.02 to $0.4 \%$ of all tumors and comprises approximately $80 \%$ of primary pleural tumors (Yang et al., 2008; Rudd, 2010). In recent years, the incidence of the disease has increased every year. Millions of people are diagnosed with MPM every year all over the globe. The incidence in Australia is four in 100,000, ranking first in the world. In China, the incidence is highest in Dayao county, Yunnan Province (Tsao et al., 2009; Gill et al., 2009). According to the latest estimate, MPM population incidence may double over the next 20 years (HopkinsDonaldson et al., 2006). Asbestos exposure history is a high-risk factor for MPM. Approximately $70-80 \%$ of MPM patients have confirmed histories of occupational exposure to asbestos (Yang et al., 2008). The prognosis of malignant mesothelioma is very poor. The average survival rate after diagnosis is only 7 to 11 months. When confirmed, most patients die within 2 years.

MPM has an insidious onset. Its clinical manifestations lack specificity and are mostly dyspnea-related. Dyspnea is associated with pleural effusion in the early stage, and with reduced chest wall activity in the later stage. With the progress of the disease and tumor metastasis, clinical manifestations include symptoms of progressive aggravated dyspnea, chest pain, and cough, which are accompanied by weight loss, fatigue, fever, and other systemic symptoms as well as pleural effusions, chest wall mass, and other signs. The above manifestations are easily confused with tuberculous pleurisy, pleural metastases, and other diseases, and are often misdiagnosed clinically.

Specific treatments of MPM remain insufficient, including surgical resection, postoperative radiotherapy, and chemotherapy along with other comprehensive treatments. However, these existing methods can only serve to improve patients' quality of life and prolong their survival time. Surgery can be used for tumor resection, which can alleviate the symptoms of patients with MPM, such as controlling local exudation, and relieving dyspnea and chest pain. Extrapleural pneumonectomy (EPP) and pleurectomy/decortications (P/D) are the two most commonly used surgical methods for MPM (Vogelzang et al., 2003; Flores, 2009). Due to difficulties in diagnosis, the extent of the disease is often relatively large once the disease is confirmed. A single surgical treatment of MPM, with either EPP or P/D, generally does not markedly prolong survival time of patients. Because MPM is not sensitive to chemotherapy drugs, chemotherapy can be used alone or in combination with surgery to cure MPM. Pemetrexed in combination with cisplatin is the recommended first-line chemotherapy method for advanced MPM. In addition to systemic chemotherapy, intrathoracic chemotherapy is often used in the treatment of MPM to control pleural effusion. MPM shows general radiation sensitivity, and thus the effect of radiotherapy is not satisfactory. Fractionated improved enhanced radiotherapy is relatively more effective than ordinary radiotherapy (Baldini, 2009).

In order to develop better treatment methods for MPM, 23 cases of MPM were cured mainly by surgery in the Thoracic Surgery Department of our hospital from January 2000 to June 2005. 


\section{MATERIAL AND METHODS}

\section{General data of patients}

Twenty-three cases of MPM were cured by surgery in the Thoracic Surgery Department of the First Affiliated Hospital of Kunming Medical University from January 2000 to June 2005, including 14 males and 9 females. They were aged from 14 to 76 years and the median age was 43 years. All patients were preoperatively examined with chest and abdominal computed tomography to exclude invasion and metastasis of the contralateral thorax and peritoneal cavity. Body conditions of patients were generally good (Karnofsky performance scale $>70$ ). No serious heart, lung, liver, or kidney dysfunction was observed. Blood routine was in the normal range and blood clotting was normal. All cases were confirmed to be MPM based on postoperative pathology, including 11 cases of epithelial-type MPM, three cases of sarcoma-type MPM, and nine cases of mixed-type MPM. The postoperative tumor-node-metastasis (TNM) stage (according to the mesothelioma-staging system modified by the Institute of International Mesothelioma (Kent et al., 2008; Salahudeen et al., 2009) was as follows: 12 cases in stage I (six cases of $\mathrm{T}_{1} \mathrm{~N}_{0} \mathrm{M}_{0}$ and six cases of $\mathrm{T}_{2} \mathrm{~N}_{0} \mathrm{M}_{0}$ ); eight cases in stage II (five cases of $T_{1} N_{1} M_{0}$ and three cases of $T_{2} N_{1} M_{0}$ ); three cases in stage III (two cases of $T_{3} N_{1} M_{0}$ and one case of $\mathrm{T}_{2} \mathrm{~N}_{2} \mathrm{M}_{0}$ ).

\section{Treatment methods}

All 23 patients of this group were treated by surgery after detailed inspection. Most (15 cases) were treated by pleurectomy. Four cases were treated by local tumor extensive resection, one case was treated by tumor + part of chest wall resection, one case was treated by pleura decortication + right middle lobe resection, and two cases were treated by palliative resection. In surgery, the parietal pleura, and part of the pericardium and diaphragm were resected. Lung tissue was reserved. Involved visceral pleura were stripped as much as possible. Miliary lesions scattered on the visceral pleura, which cannot be extensively stripped, were burnt with emphasis by electric knife. Meanwhile, mediastinal lymph nodes were cleaned. The pericardia and diaphragms of six patients with large coloboma were repaired with dacron, whereas those of others were sutured directly. Closed thoracic drainage tubes were placed conventionally before the chest was closed (the 6th or 7th intercostal between the middle axillary line and posterior axillary line on the left side, the 5th or 6th intercostal between the middle axillary line and posterior axillary line on the right side). None of the patients died within the first 15 days following surgery.

Once pleural fluid drainage was less than $50 \mathrm{~mL} /$ day, approximately $48-72 \mathrm{~h}$ after surgery, chest X-rays were reviewed. Lung reexpansion was good, and infusion chemotherapy was initiated. First, $10 \mathrm{~mL} \mathrm{2 \%} \mathrm{lidocaine} \mathrm{with} 10 \mathrm{mg}$ dexamethasone was injected from the chest tube. Patients were told to gently turn over and change position. Cisplatin, at a dose of $60 \mathrm{mg} / \mathrm{m}^{2}$, was dissolved in $50 \mathrm{~mL} 5 \%$ glucose solution, and was then injected into the pleural cavity from the chest tube. The drainage tube was removed after injection and the wound was closed. Patients were then told to turn over and change position to allow the liquid medicine to make extensive contact with pleura. If any symptom of pleural irritation was obvious, 50-100 $\mathrm{mg}$ pethidine hydrochloride was intramuscularly injected temporarily. 
Four to eight weeks after surgery, combination chemotherapy of paclitaxel and carboplatin was initiated for two cycles. Unilateral thoracic radiotherapy with a total dose of 55 Gy was administered after chemotherapy for 5 to 6 weeks in all. Then, combination chemotherapy of paclitaxel and carboplatin was administered for two cycles. After the triple regimen, patients were followed up every 3 to 4 months in order to detect relapses and prepare for retreatment.

\section{Follow-up}

All patients were followed up conventionally after surgery. Outpatient follow-up was conducted according to standard procedures. The deadline was June 2007. The follow-up period ranged from 2 to 5 years. Three patients were lost midway through the follow-up period. Lost to follow-up time was recorded as the time of death. The follow-up rate was $87.0 \%$.

\section{Statistical analysis}

The SPSS12.0 statistical software package was used. Follow-up data were tested with the log-rank test. Single factor analysis of survival was carried out and a survival curve was constructed.

\section{RESULTS}

The median survival time of all patients was 15 months (range: 3 to 89 months). The $1-, 2-$, and 3-year survival rates were $69.6,43.5$, and $13.0 \%$, respectively. The 1-year survival rates of patients in stage I, stage II, and stage III were 83.3, 62.5, and $33.3 \%$, respectively. The 2 -year survival rates were $50,37.5$, and $33.3 \%$, respectively, and the 3 -year survival rates were 34,23 , and $0 \%$, respectively (Figure 1).

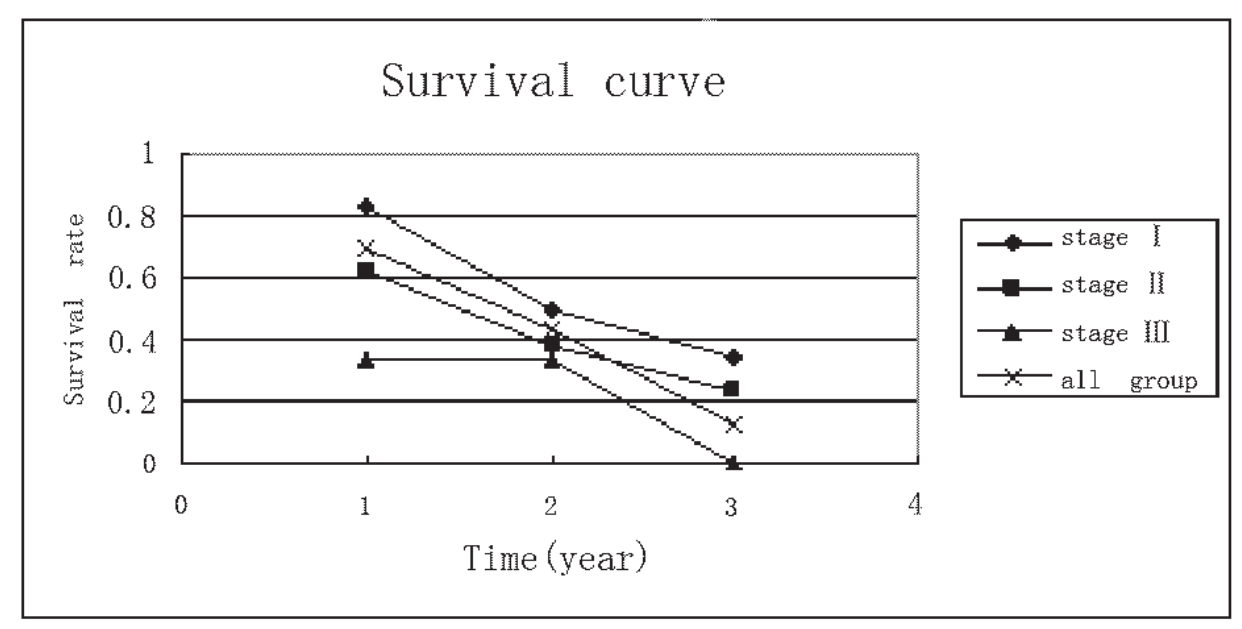

Figure 1. Survival curve of all the enrolled patiens in this study. 


\section{DISCUSSION}

Mesothelioma is a kind of epithelial or sarcoma tumor that is formed by multi-directionally differentiated mesothelial and subserosal cells. It is divided into a localized type and a diffuse type. The localized type mainly invades local pleura and is relatively rare. The diffuse type is relatively common, and occurs in any part of the splanchnic layer, including the interlobar fissure, pericardium, and lower lobe of the lung. It has a tendency to spread and invade downward (to the peritoneal cavity). Under ordinary optical microscope, MPM can be divided into epithelial type (approximately 60\%), sarcoma type (approximately 10\%), and mixed type (approximately 30\%) on the basis of histological features. Prognosis of epithelial type MPM is relatively the best (median survival time of approximately 14 months). Prognosis of sarcoma type is the worst (median survival time of approximately 7 months). Prognosis of mixed type is intermediate (Antman et al., 1983). The biological behavior is mostly local infiltration. Even untreated patients in advanced stages rarely show distant bloodline transfer (Baldini et al., 1997). Therefore, the control of chest lesions is very important.

At present, a relatively mature trimodality therapy schedule developed by the Thoracic Surgery Department of Brigham and Women's Hospital in Massachusetts, USA (Antman et al., 1989; Jaklitsch et al., 2001) is generally adopted. First, cytoreductive surgery is received. Four to eight months later, combination chemotherapy of paclitaxel and carboplatin is received for two cycles. Then, unilateral thoracic radiotherapy with a total dose of 55 Gy is received for 5 to 6 weeks in all. During radiotherapy, $60 \mathrm{mg} / \mathrm{m}^{2}$ paclitaxel single-agent chemotherapy is received once a week. Combination chemotherapy of paclitaxel and carboplatin is received for two cycles after radiotherapy.

Complete surgical resection in the early stage is the basis for successful treatment. Localized mesothelioma can be resected by surgery, and most will not recur after local resection. Due to extensive lesions and pleural cavity exudate, diffuse malignant mesothelioma is hard to be cured radically. The incidence of surgical complications and mortality are high. Therefore, radical surgery is not recommended. Comprehensive treatments of offload surgery and other methods are also available. However, even patients with MPM in progressive stages can improve their quality of life by surgery and create conditions for radiotherapy to prolong their lives.

EPP and pleural resection are common surgical treatments of MPM at present (Ettinger et al., 2010); however, which of the two strategies is better remains uncertain. It is generally believed that they show no significant difference with respect to long-term survival effects (Shi et al., 2002). As long as the right choices are made, both surgical treatments can achieve complete resection of macroscopic tumors that are seen with the naked eye. Generally, pleural resection can reserve lung tissue, which allows patients to recover quickly after surgery, thus increasing the benefits of radiotherapy and chemotherapy. However, the lesions scattered in the visceral pleura are difficult to strip completely. Wide-range burning with an electric knife can still achieve certain effects. Of course, for visceral pleura extensive involvement, pleural cavity atresia, lung tissue involvement, young patients, and patients in good body condition, the best method remains extrapleural pneumonectomy.

Most MPM types show diffusibility. Compared to other thoracic tumors, MPM has a larger radiation field. Furthermore, because MPM is adjacent to vital organs, the exposure dose is affected. Single radiotherapy often does not yield good results. A survey conducted 
at the Boston United Radiotherapy Center (Ball and Cruickshank, 1990) showed that of 23 patients with MPM who received $<40$ Gy radiotherapy, only one patient's condition was remitted. Of the six patients that received $>60$ Gy radiotherapy, four showed remission. Some patients with larger tumor burdens might need an exposure dose up to $60 \mathrm{~Gy}$. Currently, radical exposure doses are mostly more than $50 \mathrm{~Gy}$, which often leads to radiation esophagitis, pneumonia, hepatitis, myocarditis, and other complications. Therefore, assessments should be done before radiotherapy and, if necessary, protective measures should be taken.

MPM shows a natural resistance to chemotherapeutic drugs (Tsiouris and Walesby, 2007). Doxorubicin-oriented cytotoxic drugs were originally used. Since 1985, the CAP scheme has been the first-line chemotherapy regimen, and is still used today. In 1995, combination chemotherapy of paclitaxel and carboplatin became the first-line chemotherapy scheme for MPM (Pass et al., 1997).

Pemetrexed (Alimta) is a new kind of multi-target anti-folate drug. It acts on several enzymes in folate-dependent metabolic pathways, including thymidylate synthase (TS), dihydrofolate reductase (DHFR), and glycinamide ribonucleotide transformylase (GARFT), among others, which results in inhibition of the synthesis of purines and pyrimidines. Consequently, the cell cycle stagnates in the $\mathrm{S}$ phase, thus promoting apoptosis of tumor cells to achieve anti-cancer effects (Hanauske et al., 2001). In February 2004, pemetrexed was approved by the U.S. Food and Drug Administration. Combined with platinum agents, it was used as first-line chemotherapy drugs of MPM (Hazarika et al., 2004) to cure unresectable MPM. However, due to its high cost, pemetrexed is not yet widely used in China.

Pleural adhesion is one of the main treatments for pleural mesothelioma (Gao et al., 2005; Hassan and Ho, 2008; Vachani et al., 2010). Intrapleural perfusion of cisplatin for intrapleural chemotherapy can increase local drug concentrations and reduce systemic side effects. Moreover, in addition to its antitumor function, cis-platinum also has obvious chemical inflammatory effects. Therefore, cis-platinum has both local chemotherapy and pleural adhesion functions. Remarkably, the amount of pleural effusion before medication directly affects the effect of chemotherapy and pleural adhesions. Therefore, before infusion of cisplatin, pleural effusion should be drained as much as possible to achieve good therapeutic results.

New treatments, such as mesothelin-targeted gene therapy and SV40 vaccine-applied gene therapy, are currently in clinical trial stages.

Although MPM is a highly malignant tumor, metastasis rarely occurs because its main growth pattern is local infiltration. As long as patients' conditions permit it, surgical treatment should be adopted actively so as to reduce tumor cells. Certain therapeutic effects can be achieved with postoperative radiotherapy and chemotherapy, whether or not MPM is cured radically, survival time can be prolonged, and prognosis, as well as life quality, can be improved.

\section{REFERENCES}

Antman KH, Corson JM, Li FP, Greenberger J, et al. (1983). Malignant mesothelioma following radiation exposure. $J$. Clin. Oncol. 1: 695-700.

Antman KH, Pass HI and Recht A (1989). Benign and Malignant Mesothelioma. In: Cancer, Principles and Practice of Oncology. 3rd edn. Lippincott, Philadelphia, 1399-1417.

Baldini EH (2009). Radiation therapy options for malignant pleural mesothelioma. Semin. Thorac. Cardiovasc. Surg. 21: $159-163$.

Baldini EH, Recht A, Strauss GM, DeCamp MM Jr, et al. (1997). Patterns of failure after trimodality therapy for malignant pleural mesothelioma. Ann. Thorac. Surg. 63: 334-338. 
Ball DL and Cruickshank DG (1990). The treatment of malignant mesothelioma of the pleura: review of a 5-year experience, with special reference to radiotherapy. Am. J. Clin. Oncol. 13: 4-9.

Ettinger DS, Akerley W and Bepler G (2010). Malignant Pleural Mesotheliorna V.1.2010. NCCN Clinical Practice Guidelines in Oncology, Fort Washington.

Flores RM (2009). Surgical options in malignant pleural mesothelioma: extrapleural pneumonectomy or pleurectomy/ decortication. Semin. Thorac. Cardiovasc. Surg. 21: 149-153.

Gao XL, Chen ZX and Guo J (2005). Medical thoracoscopy for diagnosis and treatment of pleural mesothelioma. China J. Endosc. 11: 30-32.

Gill RR, Gerbaudo VH, Sugarbaker DJ and Hatabu H (2009). Current trends in radiologic management of malignant pleural mesothelioma. Semin. Thorac. Cardiovasc. Surg. 21: 111-120.

Hanauske AR, Chen V, Paoletti P and Niyikiza C (2001). Pemetrexed disodium: a novel antifolate clinically active against multiple solid tumors. Oncologist 6: 363-373.

Hassan R and Ho M (2008). Mesothelin targeted cancer immunotherapy. Eur. J. Cancer 44: 46-53.

Hazarika M, White RM, Johnson JR and Pazdur R (2004). FDA drug approval summaries: pemetrexed (Alimta). Oncologist 9: 482-488.

Hopkins-Donaldson S, Belyanskaya LL, Simoes-Wust AP, Sigrist B, et al. (2006). p53-induced apoptosis occurs in the absence of p14(ARF) in malignant pleural mesothelioma. Neoplasia 8: 551-559.

Jaklitsch MT, Grondin SC and Sugarbaker DJ (2001). Treatment of malignant mesothelioma. World J. Surg. 25: 210-217.

Kent M, Rice D and Flores R (2008). Diagnosis, staging, and surgical treatment of malignant pleural mesothelioma. Curr. Treat. Options. Oncol. 9: 158-170.

Pass HI, Kranda K, Temeck BK, Feuerstein I, et al. (1997). Surgically debulked malignant pleural mesothelioma: results and prognostic factors. Ann. Surg. Oncol. 4: 215-222.

Rudd RM (2010). Malignant mesothelioma. Br. Med. Bull. 93: 105-123.

Salahudeen HM, Hoey ET, Robertson RJ and Darby MJ (2009). CT appearances of pleural tumours. Clin. Radiol. 64: 918-930.

Shi JX, Gao CX and Chen W (2002). Surgical therapy for malignant pleural mesothelioma. Tumor 22: 134-135.

Tsao AS, Wistuba I, Roth JA and Kindler HL (2009). Malignant pleural mesothelioma. J. Clin. Oncol. 27: 2081-2090.

Tsiouris A and Walesby RK (2007). Malignant pleural mesothelioma: current concepts in treatment. Nat. Clin. Pract. Oncol. 4: 344-352.

Vachani A, Moon E, Wakeam E and Albelda SM (2010). Gene therapy for mesothelioma and lung cancer. Am. J. Respir. Cell Mol. Biol. 42: 385-393.

Vogelzang NJ, Rusthoven JJ, Symanowski J, Denham C, et al. (2003). Phase III study of pemetrexed in combination with cisplatin versus cisplatin alone in patients with malignant pleural mesothelioma. J. Clin. Oncol. 21: 2636-2644.

Yang H, Testa JR and Carbone M (2008). Mesothelioma epidemiology, carcinogenesis, and pathogenesis. Curr. Treat. Options Oncol. 9: 147-157. 